\title{
Resting-state fMRI as a biomarker for Alzheimer's disease?
}

\author{
Jessica S Damoiseaux* \\ See related review by Vemuri et al., http://alzres.com/content/4/1/2
}

\begin{abstract}
Previous work indicates that resting-state functional magnetic resonance imaging (fMRI) is sensitive to functional brain changes related to Alzheimer's disease (AD) pathology across the clinical spectrum. Crosssectional studies have found functional connectivity differences in the brain's default mode network in aging, mild cognitive impairment, and AD. In addition, two recent longitudinal studies have shown that functional connectivity changes track AD progression. This earlier work suggests that resting-state fMRI may be a promising biomarker for AD. However, some key issues still need to be addressed before resting-state fMRI can be successfully applied clinically. In a previous issue of Alzheimer's Research \& Therapy, Vemuri and colleagues discuss the use of resting-state fMRI in the study of AD. In this commentary, I will highlight and expand upon some of their main conclusions.
\end{abstract}

Resting-state functional magnetic resonance imaging (fMRI), a technique used to image intrinsic functional brain connectivity, is considered a promising biomarker for Alzheimer's disease (AD) as functional brain changes are thought to precede structural brain changes. Previous work has shown that resting-state functional connectivity is sensitive to functional brain changes related to $A D$ pathology across the clinical spectrum. Resting-state functional connectivity changes within the default mode network, originally identified by Raichle and colleagues [1], have been observed in healthy aging [2,3], mild cognitive impairment, a prodromal stage of AD [4-6], and $\mathrm{AD}$ [7-9].

In a review in a previous issue of Alzheimer's Research $\mathcal{E}$ Therapy, Vemuri and colleagues [10] point out that the

${ }^{*}$ Correspondence: jeske@stanford.edu

Functional Imaging in Neuropsychiatric Disorders Lab, Department of Neurology and Neurological Sciences, Stanford University School of Medicine, 780 Welch Road, Suite 105, Palo Alto, CA 94304, USA most consistent finding across previous resting-state fMRI studies of $A D$ is decreased functional connectivity in $\mathrm{AD}$ patients versus healthy older controls in a posterior default mode network region composed of the precuneus and posterior cingulate cortex. The review furthermore addresses the recent findings of several additional studies showing increased connectivity in $\mathrm{AD}$ patients versus healthy older controls in frontal default mode regions. This increase in functional connectivity could be interpreted as a possible compensatory mechanism (within the default mode network) that is triggered by the functional loss in posterior brain regions. This process is similar to the increased activity that has been observed in frontal brain regions in task-related fMRI studies of aging and that has been associated with better performance [11]. Recently, two longitudinal resting-state studies that support this idea of a compensatory mechanism in the progression of $\mathrm{AD}$ were published [12,13]. In patients with amnestic mild cognitive impairment, functional connectivity within posterior default mode brain regions was increased in comparison with healthy controls; however, at follow-up, connectivity in these areas was decreased and connectivity in frontal default mode regions was increased [12]. In patients with $\mathrm{AD}$, connectivity at baseline was decreased in the posterior default mode areas and increased in frontal regions in comparison with healthy controls. However, at follow-up, patients showed decreased connectivity throughout the entire default mode network [13]. These results suggest that, within the default mode network, hyper-connectivity precedes hypo-connectivity of a brain region, and this may signal the early phase of brain dysfunction. Note that the observed connectivity changes follow the trajectory of neuropathology as previously described by Braak and Braak [14], which affects the medial temporal lobe first, followed by posterolateral cortical regions and, in the latest stages, the frontal cortex. These previous findings, which outline the functional connectivity changes as the disease progresses, support the potential of resting-state fMRI as a biomarker to uncover signs of incipient AD.

In our longitudinal study [13], most brain clusters that over time showed a decrease in functional connectivity in 
patients showed an increase in controls. Though very tentative, this finding could support the theory that this process of hyper-connectivity, which reflects functional compensation, already starts in normal aging. In their review, Vemuri and colleagues mention that, even though changes in the default mode network have been observed in normal aging, the age effect is accelerated in $\mathrm{AD}$ [15]. A critical question that needs to be addressed is whether we will be able to distinguish early abnormal connectivity changes from normal age-related changes. Therefore, an avenue for future research is to study longitudinal functional connectivity changes in normal older adults and investigate the effects of certain risk factors for AD, such as genetics, gender, cognitive function, and physical fitness, on these changes.

However, before resting-state functional connectivity can be implemented as a biomarker for $\mathrm{AD}$, there are still some additional issues that need to be addressed. So far, the ability to meaningfully use fMRI data (that is, data of resting-state as well as task-related fMRI) on a singlesubject level has been very limited. Changes in the acquisition of fMRI data could potentially improve the signal-to-noise ratio of the data and thus increase the power of our statistical analyses. In addition, changes in the analysis methods may further improve the sensitivity of the measurements. One example of such an improvement in the analysis methods is the creation and subsequent use of a functional brain atlas instead of a structural brain atlas for assessing functional connectivity strength. Moreover, there is a lot of variability between individual patients and this possibly also explains why it has been difficult to obtain an adequate level of sensitivity and specificity by using classification procedures. By following the functional connectivity changes in disease progression and additionally investigating the very early (that is, latent) stage of $\mathrm{AD}$, we may be able to understand the changes that take place during the entire disease trajectory. This knowledge may help us improve our ability to appropriately classify new subjects and ultimately allow us to use resting-state functional connectivity as a biomarker for AD.

Abbreviations

AD, Alzheimer's disease; fMRI, functional magnetic resonance imaging.
Competing interests

The author declares that she has no competing interests.

Published: 15 March 2012

\section{References}

1. Raichle ME, MacLeod AM, Snyder AZ, Powers WJ, Gusnard DA, Shulman GL: A default mode of brain function. Proc Natl Acad Sci U SA 2001, 98:676-682.

2. Damoiseaux JS, Beckmann CF, Arigita EJ, Barkhof F, Scheltens P, Stam CJ, Smith SM, Rombouts SA: Reduced resting-state brain activity in the "default network" in normal aging. Cereb Cortex 2008, 18:1856-1864.

3. Andrews-Hanna J, Snyder A, Vincent J, Lustig C, Head D, Raichle M, Buckner R: Disruption of large-scale brain systems in advanced aging. Neuron 2007, 56:924-935.

4. Bai F, Watson DR, Yu H, Shi Y, Yuan Y, Zhang Z: Abnormal resting-state functional connectivity of posterior cingulate cortex in amnestic type mild cognitive impairment. Brain Res 2009, 1302:167-174.

5. Petrella JR, Sheldon FC, Prince SE, Calhoun VD, Doraiswamy PM: Default mode network connectivity in stable vs progressive mild cognitive impairment. Neurology 2011, 76:511-517.

6. Sorg C, Riedl V, Mühlau M, Calhoun V, Eichele T, Läer L, Drzezga A, Förstl H, Kurz A, Zimmer C, Wohlschläger A: Selective changes of resting-state networks in individuals at risk for Alzheimer's disease. Proc Natl Acad Sci USA 2007, 104:18760-18765.

7. Greicius MD, Srivastava G, Reiss AL, Menon V: Default-mode network activity distinguishes Alzheimer's disease from healthy aging: evidence from functional MRI. Proc Natl Acad Sci U S A 2004, 101:4637-4642.

8. Wang K, Liang M, Wang L, Tian L, Zhang X, Li K, Jiang T: Altered functional connectivity in early Alzheimer's disease: a resting-state fMRI study. Hum Brain Mapp 2007, 28:967-978.

9. Zhang HY, Wang SJ, Xing J, Liu B, Ma ZL, Yang M, Zhang ZJ, Teng GJ: Detection of PCC functional connectivity characteristics in resting-state fMRI in mild Alzheimer's disease. Behav Brain Res 2009, 197:103-108.

10. Vemuri P, Jones DT, Jack CR Jr.: Resting state functional MRI in Alzheimer's Disease. Alzheimers Res Ther 2012, 4:2.

11. Cabeza R, Anderson ND, Locantore JK, McIntosh AR: Aging gracefully: compensatory brain activity in high-performing older adults. Neuroimage 2002, 17:1394-1402.

12. Bai F, Watson DR, Shi Y, Wang Y, Yue C, Teng Y, Wu D, Yuan Y, Zhang Z: Specifically progressive deficits of brain functional marker in amnestic type mild cognitive impairment. PLOS ONE 2011, 6:e24271.

13. Damoiseaux JS, Prater KE, Miller BL, Greicius MD: Functional connectivity tracks clinical deterioration in Alzheimer's disease. Neurobiol Aging 2012, 33:828.e19-30.

14. Braak H, Braak E: Neuropathological stageing of Alzheimer-related changes. Acta Neuropathol 1991, 82:239-259.

15. Jones DT, Machulda MM, Vemuri P, McDade EM, Zeng G, Senjem ML, Gunter $J$, Przybelski SA, Avula RT, Knopman DS, Boeve BF, Petersen RC, Jack CR Jr:: Age-related changes in the default mode network are more advanced in Alzheimer disease. Neurology 2011, 77:1524-1531.

doi:10.1186/alzrt106

Cite this article as: Damoiseaux JS: Resting-state $\mathrm{fMRI}$ as a biomarker for Alzheimer's disease? Alzheimer's Research \& Therapy 2012, 4:8. 